\title{
Myocardial adaptation after surgical therapy differs for aortic valve stenosis and hypertrophic obstructive cardiomyopathy
}

\author{
Rahana Y. Parbhudayal ${ }^{1,2,10}$. Ahmet Güçlü̈ · Alwin Zweerink ${ }^{1}$ P. Stefan Biesbroek ${ }^{1}$. Pierre Croisille ${ }^{4}$. \\ Patrick Clarysse ${ }^{5} \cdot$ Michelle Michels $^{6} \cdot$ Wim Stooker $^{7} \cdot$ Alexander B. A. Vonk ${ }^{8} \cdot$ Peter M. van der Ven ${ }^{9}$. \\ Albert C. van Rossum ${ }^{1}$. Jolanda van der Velden ${ }^{2,10} \cdot$ Robin Nijveldt $^{1}$
}

Received: 6 December 2018 / Accepted: 12 February 2019 / Published online: 1 March 2019

(c) The Author(s) 2019

\begin{abstract}
Surgical therapies in aortic valve stenosis (AVS) and hypertrophic obstructive cardiomyopathy (HOCM) aim to relief intraventricular pressure overload and improve clinical outcome. It is currently unknown to what extent myocardial adaptation concurs with restoration of intraventricular pressures, and whether this is similar in both patient groups. The aim of this study was to investigate changes in myocardial adaptation after surgical therapies for AVS and HOCM. Ten AVS and ten HOCM patients were enrolled and underwent cardiac magnetic resonance cine imaging and myocardial tagging prior to, and 4 months after aortic valve replacement (AVR) and septal myectomy, respectively. Global left ventricular (LV) analyses were derived from cine images. Circumferential strain was assessed from myocardial tagging images at the septal and lateral wall of the mid ventricle. Pressure gradients significantly decreased in both AVS and HOCM after surgery $(p<0.01)$, with a concomitant decrease in left atrial volume $(p<0.05)$ suggesting lower diastolic filling pressures. Also, LV volumes, mass and septal wall thickness decreased in both, but to a larger extent in AVS than in HOCM patients. AVR improved wall thickening $(p<0.05)$ and did not change systolic strain rate. Myectomy did not affect wall thickening and reduced septal systolic strain rate $(p=0.03)$. Both AVR and myectomy induced positive structural remodeling in line with a reduction of pressure overload. A concomitant recovery in systolic function however was found in AVR only. The systolic functional deterioration in HOCM patients seems to be inherent to myectomy and the ongoing and irreversible disease.
\end{abstract}

Keywords Aortic valve stenosis $\cdot$ Hypertrophic obstructive cardiomyopathy $\cdot$ Magnetic resonance imaging $\cdot$ Cardiac remodeling

Electronic supplementary material The online version of this article (https://doi.org/10.1007/s10554-019-01563-3) contains supplementary material, which is available to authorized users.

Robin Nijveldt

robin@ nijveldt.net

1 Department of Cardiology, Amsterdam UMC, Vrije Universiteit Amsterdam, Amsterdam Cardiovascular Sciences, De Boelelaan 1117, 1081 HV, Amsterdam, The Netherlands

2 Department of Physiology, Amsterdam UMC, Vrije Universiteit Amsterdam, Amsterdam Cardiovascular Sciences, Amsterdam, The Netherlands

3 Department of Cardiology, Isala Klinieken, Zwolle, The Netherlands

4 Univ Lyon, UJM-Saint-Etienne, INSA, CNRS UMR 5520, Inserm U1206, Creatis, 42023 Sint-Etienne, France
Abbreviations

AVS Aortic valve stenosis

AVR Aortic valve replacement

HOCM Hypertrophic obstructive cardiomyopathy

5 Univ Lyon, INSA-Lyon, Université Claude Bernard Lyon 1, UJM-Saint Etienne, CNRS, Inserm, Creatis UMR 5220, U1206, 69621 Lyon, France

6 Department of Cardiology, Erasmus Medical Center, Rotterdam, The Netherlands

7 Department of Cardiothoracic Surgery, Onze Lieve Vrouwe Gasthuis, Amsterdam, The Netherlands

8 Department of Cardiothoracic Surgery, VU University Medical Center Amsterdam, Amsterdam, The Netherlands

9 Department of Epidemiology and Biostatistics, VU University Medical Center, Amsterdam, The Netherlands

10 The Netherlands Heart Institute, Utrecht, The Netherlands 


\section{Left ventricular \\ LVH Left ventricular hypertrophy \\ LVOT Left ventricular outflow tract \\ CMR Cardiovascular magnetic resonance \\ EDWT End-diastolic wall thickness \\ bSSFP Balanced steady-state free precession}

\section{Introduction}

Left ventricular hypertrophy (LVH) is a common finding in clinical practice and is associated with morbidity and mortality. LVH can be detected in acquired and genetic cardiac diseases. The most common cause for acquired LVH is in aortic valve stenosis (AVS). In response to systolic pressure overload, the myocardium hypertrophies in an attempt to normalize increased wall stress [1]. Patients with severe AVS accompanied with LVH have an increased risk to develop heart failure in the future [2]. Aortic valve replacement (AVR) therapy is recommended in all symptomatic AVS patients, and has been shown to improve left ventricular ejection fraction (LVEF), exercise capacity, and mortality [3]. Considering genetic cardiomyopathies, hypertrophic obstructive cardiomyopathy (HOCM) is the most common cause for LVH with a prevalence ranging from 1:200 to 1:500 [4]. Sarcomeric mutations affect functional properties of the sarcomeres [5] and impair energy metabolism, leading to $\mathrm{LVH}$, most often asymmetric [6, 7]. This asymmetric hypertrophy in combination with systolic anterior motion of the mitral valve can cause left ventricular outflow tract (LVOT) obstruction, leading to heterogeneous symptoms, varying from angina and syncope, to congestive heart failure and sudden cardiac death [8]. The surgical treatment for LVOT obstruction is septal myectomy, which reduces the risk for sudden cardiac death and normalizes left ventricular (LV) pressures [9]. Previous studies have investigated the effect of AVR and septal myectomy on the myocardium separately demonstrating a reduction in intraventricular pressures with subsequent improvement in clinical symptoms and outcome [10-13]. However, it is currently unclear to what extent myocardial structural and functional recovery concurs with restoration of intraventricular pressures, and whether this is comparable in patient groups with similar concentric hypertrophic remodeling, but a different cause (i.e. aortic stenosis vs. genetic). In the current study, we use cardiac magnetic resonance (CMR) imaging to accurately assess structural changes and myocardial function after surgery for AVS and HOCM, and compare this change to a group of healthy controls. We hypothesize that surgical therapies will improve myocardial function in both AVS and HOCM patients.

\section{Methods}

The study protocol was in agreement with the principles outlined in the Declaration of Helsinki and was approved by the Medical Ethics Review committees of the participating hospitals (VU University Medical Center and Onze Lieve Vrouwe Gasthuis in Amsterdam, The Netherlands). All participants gave written informed consent prior to inclusion.

Ten AVS patients eligible for AVR therapy and ten HOCM patients eligible for septal myectomy, were prospectively enrolled in the study between October 2011 and November 2015, as described previously [14]. Inclusion criteria for AVS participants were the presence of isolated AVS with a peak transvalvular pressure gradient of $>50 \mathrm{mmHg}$, and an aortic valve area $<1 \mathrm{~cm}^{2}$, according to the American Society of Echocardiographic guidelines [15]. Inclusion criteria for HOCM participants were LVOT peak pressure gradient $>30 \mathrm{mmHg}$ at rest or during provocation, and presence of clinical symptoms, despite optimal medical treatment. According to the guidelines to undergo surgical intervention in AVS and HOCM patients, different cutoff gradients are advised [16, 17]. The exclusion criteria were any absolute or relative contra-indication for undergoing CMR, the presence of any significant coronary artery disease ( $>30 \%)$ and a history of hypertension in HOCM patients. LVOT gradient and peak aortic valve pressure gradient were obtained by Doppler echocardiography. Maximal exercise capacity was derived from a cyclo ergometry test when patients reached a point of exhaustion or symptom limitation, prior to, and after surgical therapy with a ramp protocol of $10-20 \mathrm{~W} \mathrm{~min}{ }^{-1}$. To compare myocardial function in AVS and HOCM patients before surgical therapy, 14 gender-matched healthy subjects were included as a control group.

All AVS and HOCM participants underwent a CMR scan, 2 weeks prior to, and 4 months after surgery. CMR was performed on a 1.5 Tesla whole body scanner (Magnetom Sonata or Avanto, Siemens, Erlangen, Germany), using a six-channel phased-array body coil. In all patients and controls, cine images were obtained using a breathhold segmented k-space balanced steady-state free precession (bSSFP) employing retrospective electrocardiographic gating, with contiguous short axis slices to cover the whole ventricle from base to apex. Ventricular volumes at end-diastole and end-systole, and mass were obtained from the cine short axis images. Left atrial (LA) volumes and emptying fraction (LAEF) were obtained from a stack of transversely oriented slices on a two-chamber view at the level of the lower leading edge of the mitral valve annulus to cover the left atrium [18]. A multiple breath-hold, retrospectively triggered bSSFP myocardial 
sinusoidal complementary tagged (CSPAMM) images were acquired to create non-invasive markers (tags) within the myocardium [19]. A midventricular short axis plane was positioned at $50 \%$ of the distance between the mitral valve annulus and the endocardial border of the apex. Additional details about the CMR acquisition are provided in the Data Supplement.

The cine images were analyzed off-line by a single investigator, using MASS analysis software (Medis medical imaging systems, v2.1, Leiden, The Netherlands). Endocardial contours were drawn at end-diastole and end-systole to calculate LV end-diastolic volume (LVEDV), LV end-systolic volume (LVESV), and LVEF. Addition of epicardial contours was used to calculate LV mass and wall thickening. Tissue tagging images were analyzed by Intag [20] software (CREATIS, Lyon, France) to quantify myocardial motion using the SinMod technique and estimate regional peak circumferential strain components (Lagrangian and systolic and diastolic strain rate, Fig. 1). Myocardial strain was measured in the mid myocardial layer which has been reported to be the most reproducible [21]. The software runs as a plug-in for OsiriX (v6.5, Pixmeo, Switzerland) [22]. Analysis of the LV was calculated according to the 17 segment AHA model [23]. To investigate regional effects after surgical treatment in both patient groups, strain analyses were derived from the septum (average of segment 8 and 9) and lateral wall (average of segment 11 and 12). Both areas were compared with each other. The lateral wall served as a remote area in which no regional treatment was performed. Because of the lack of tissue tagging on the long axis, global and regional longitudinal strain was analyzed off-line using CVi 42 software (Circle Cardiovascular Imaging, Calgery, Canada). Semiautomatic endo- and epicardial contours were drawn on the four, three and two chamber cine images. To compare septal and lateral longitudinal strain, the mean of the four septal segments of basal antero- and inferoseptal segments and four lateral segments of basal and mid antero- and inferolateral segments were calculated.

Statistical analysis was performed using SPSS software (version 22.0; SPSS, Chicago, IL, USA). All variables were visually checked for normal distribution by appreciation of the histogram with separate needs of the patient groups. All data were not normally distributed and presented by median with interquartile range and was compared to healthy controls with a Mann-Whitney-U test. Measurements prior to, and after surgical therapy were compared within groups using Wilcoxon signed-rank test. Exact Chi square test was used for categorical variables.

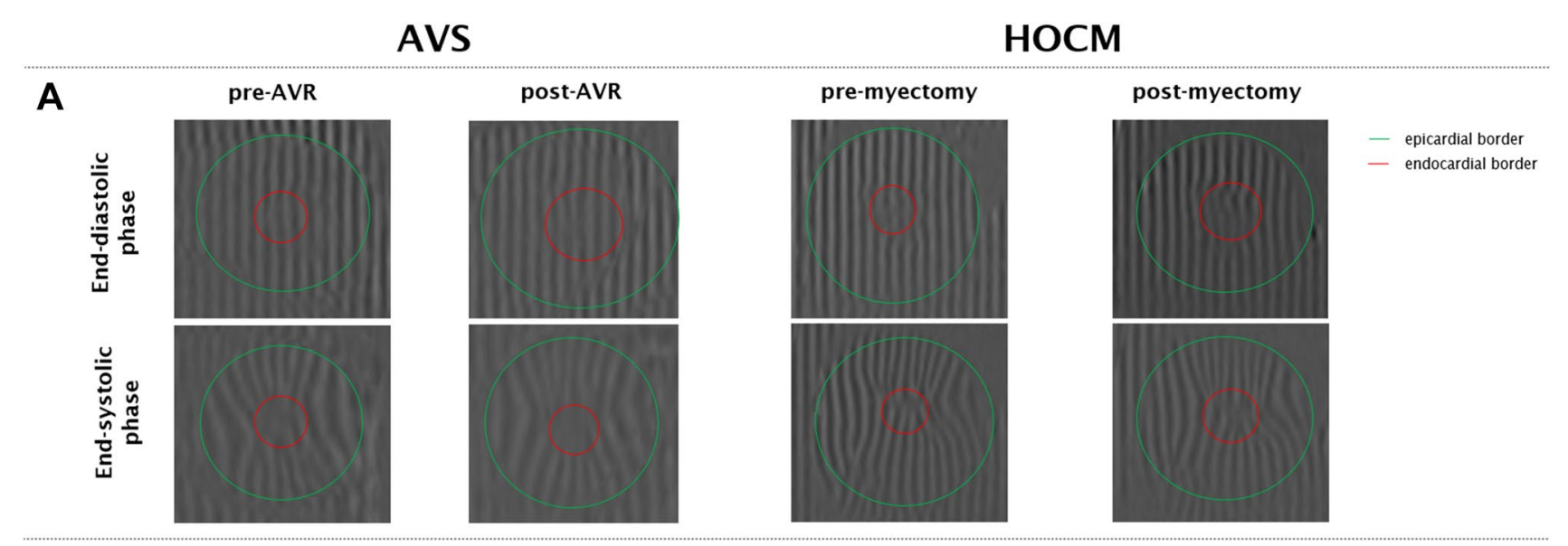

B

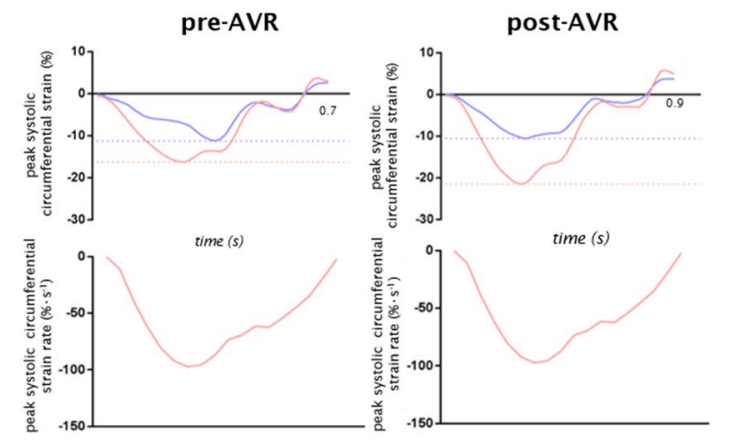

Fig. 1 Representative tagging images of a patient with AVS and HOCM with corresponding strain signals. a At end-diastole and endsystole representative images are shown for a patient with AVS (left)

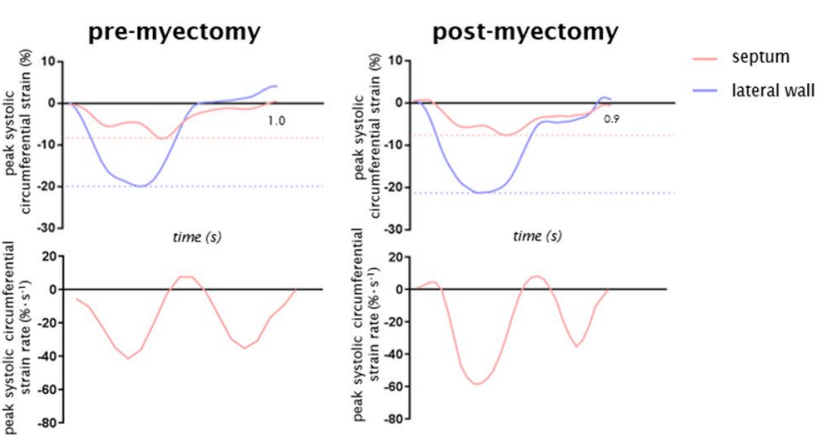

and HOCM (right) before (pre) and after (post) surgical treatment. b Corresponding peak circumferential strain and systolic circumferential strain rate curves are presented 
As three separate statistical tests were performed for each outcome measure in each group of patients (comparison of pre- and post-measurement and separate comparison of pre- and post- measurements to the control group) we used a two-sided significance level of $0.05 / 3$ to account for multiple testing.

\section{Results}

\section{Myocardial function in aortic valve stenosis before surgery}

In the AVS group none of the patients used betablockers, one patient used an ACE inhibitor and four patients used a statin before surgery. Pressure gradients were significantly higher in AVS with a concomitant higher LA volume, as presented in Tables 1 and 2. End-diastolic wall thickness (EDWT) was significantly higher compared with controls both in the septum and the lateral wall $(p<0.001)$. Except for LV mass, all global LV dimensions were comparable to controls (Table 2). Septal and lateral wall thickening were significantly lower compared with controls (Table 3, $p<0.001$ ), whereas only septal circumferential strain was significantly reduced (Table 3, $p=0.013)$. Septal systolic strain rate was reduced compared with controls $(-34[-44,-27]$ vs. $-47[-58$, -34] $\left.\% \mathrm{~s}^{-1}, p=0.005\right)$. Lateral systolic and diastolic strain rates before AVR were similar to controls (Table 3). Global longitudinal strain was similar to healthy subjects (Fig. 2).

\section{Myocardial function in hypertrophic obstructive cardiomyopathy before surgery}

In the HOCM group, eight patients used betablockers, one used an ACE inhibitor and one used a diuretic before myectomy. At baseline HOCM presented with a lower heart rate, probably due to the usage of betablockers $(p=0.003)$. Pressure gradients were significantly higher in HOCM with a concomitant higher LA volume, as listed in Tables 1 and 4. Regarding global myocardial function, HOCM patients had smaller LVESV, higher LVEF, and higher LV mass compared to controls (Table 4). Septal and lateral EDWT were higher than controls (Table 5, $p<0.001$ ). Septal wall thickening and septal circumferential strain were significantly reduced compared with controls (Table 5, $p<0.01$ ). Similar to AVS patients, septal systolic and diastolic strain rate were reduced compared with controls before myectomy $\left(-28[-44,-17]\right.$ vs. $-47[-58,-34] \% \mathrm{~s}^{-1}, p=0.005 ; 13$ $[9,33]$ vs. $36[26,39] \% \mathrm{~s}^{-1}, p=0.02$, resp.). Lateral systolic and diastolic strain rate were comparable to controls (Table 5). Interestingly, there were no differences between systolic and diastolic strain rates between AVS and HOCM patients before surgery (for both $p=0.53$ ). Global longitudinal strain was significantly affected compared with controls $(-16[-19,-14]$ vs. $-22[23,-19] \%, p=0.007$, Fig. 2).

\section{Effect on myocardial function after aortic valve replacement}

Both peak and mean transvalvular pressure gradients in AVS patients significantly decreased after surgery (from $85[72,107]$ to $23[14,32] \mathrm{mmHg}$, and from $49[42,62]$

Table 1 Baseline characteristics of controls, aortic valve stenosis and hypertrophic cardiomyopathy patients

\begin{tabular}{|c|c|c|c|c|c|c|}
\hline & Controls $(n=14)$ & $\begin{array}{l}\text { HOCM pre- } \\
\text { myectomy } \\
(\mathrm{n}=10)\end{array}$ & $\begin{array}{l}\text { AVS pre-AVR } \\
(\mathrm{n}=10)\end{array}$ & $\begin{array}{l}p \text {-value HOCM } \\
\text { pre-myectomy } \\
\text { versus controls }\end{array}$ & $\begin{array}{l}p \text {-value AVS } \\
\text { pre-AVR versus } \\
\text { controls }\end{array}$ & $\begin{array}{l}p \text {-value HOCM } \\
\text { pre-myectomy versus } \\
\text { AVS pre-AVR }\end{array}$ \\
\hline Age (years) & $50[40,57]$ & $53[47,65]$ & $59[54,68]$ & 0.37 & 0.009 & 0.14 \\
\hline $\begin{array}{l}\text { Gender (male/ } \\
\text { female) }\end{array}$ & $9 / 5$ & $6 / 4$ & $7 / 3$ & 0.83 & 0.77 & 0.64 \\
\hline $\mathrm{SBP}(\mathrm{mmHg})$ & $119[112,134]$ & $106[103,123]$ & $123[107,130]$ & 0.04 & 0.55 & 0.06 \\
\hline $\mathrm{DBP}(\mathrm{mmHg})$ & $71[65,75]$ & $59[57,70]$ & $72[60,78]$ & 0.03 & 0.89 & 0.22 \\
\hline MAP (mmHg) & $88[81,94]$ & $76[71,88]$ & $89[76,95]$ & 0.03 & 0.71 & 0.14 \\
\hline $\begin{array}{l}\text { Peak gradient Ao/ } \\
\text { LVOT }(\mathrm{mmHg})^{*}\end{array}$ & NA & $26[15,54]$ & $85[72,107]$ & NA & NA & 0.001 \\
\hline $\begin{array}{l}\text { Mean gradient Ao/ } \\
\text { LVOT }(\mathrm{mmHg})^{*}\end{array}$ & NA & $14[8,23]$ & $49[42,62]$ & NA & NA & $<0.001$ \\
\hline Heart rate $(/ \mathrm{min})$ & $69[63,78]$ & $59[57,61]$ & $68[63,70]$ & 0.003 & 0.403 & 0.005 \\
\hline
\end{tabular}

Data is presented as median and interquartile range

Ao aortic, $A V S$ aortic valve stenosis, $D B P$ diastolic blood pressure, $H O C M$ hypertrophic obstructive cardiomyopathy, $L V O T$ left ventricular outflow tract, $M A P$ mean arterial pressure, $N A$ not applicable, $P O S T$ after surgical therapy, $P R E$ before surgical therapy, $S B P$ systolic blood pressure

*Measured by Doppler echocardiography 
Table 2 Aortic valve stenosis before (PRE) and after (POST) aortic valve replacement: global characteristics

\begin{tabular}{|c|c|c|c|c|c|c|}
\hline & Controls $(n=14)$ & $\begin{array}{l}\text { AVS pre-AVR } \\
(\mathrm{n}=10)\end{array}$ & $\begin{array}{l}p \text {-value controls } \\
\text { versus AVS pre- } \\
\text { AVR }\end{array}$ & $\begin{array}{l}\text { AVS post-AVR } \\
(\mathrm{n}=10)\end{array}$ & $\begin{array}{l}p \text {-value AVS pre- } \\
\text { AVR versus AVS } \\
\text { post-AVR }\end{array}$ & $\begin{array}{l}p \text {-value AVS } \\
\text { post-AVR versus } \\
\text { controls }\end{array}$ \\
\hline \multicolumn{7}{|c|}{ Global LV characteristics } \\
\hline $\operatorname{LV} \operatorname{EDV}\left(\mathrm{ml} \mathrm{m}^{-2}\right)$ & $90[81,104]$ & $101[84,118]$ & 0.29 & $84[74,102]$ & 0.01 & 0.59 \\
\hline $\operatorname{LV} \operatorname{ESV}\left(\mathrm{ml} \mathrm{m}^{-2}\right)$ & $36[26,40]$ & $40[29,55]$ & 0.29 & $31[26,39]$ & 0.01 & 0.55 \\
\hline LVEF (\%) & $61[57,66]$ & $59[52,64]$ & 0.37 & $63[59,66]$ & 0.29 & 0.67 \\
\hline $\mathrm{SV}(\mathrm{ml})$ & $114[101,124]$ & $111[97,128]$ & 0.89 & $106[86,127]$ & 0.17 & 0.63 \\
\hline $\mathrm{CO}\left(\mathrm{L} \mathrm{min}{ }^{-1}\right)$ & $7.9[6.5,8.8]$ & $8.0[7,10.1]$ & 0.47 & $7.1[6.2,8.2]$ & 0.01 & 0.29 \\
\hline $\operatorname{LVM}\left(\mathrm{g} \mathrm{m}^{-2}\right)$ & $50[44,54]$ & $94[79,119]$ & $<0.001$ & $72[59,89]$ & 0.005 & $<0.001$ \\
\hline LGE mass (\%) LV & 0 & $0[0,1.4]$ & NA & $0[0,1.4]$ & 0.99 & NA \\
\hline \multicolumn{7}{|l|}{ LA characteristics } \\
\hline $\begin{array}{l}\text { LA volume } \\
\left(\mathrm{ml} \mathrm{m}^{-2}\right)\end{array}$ & $45[42,50]$ & $60[53,66]$ & $<0.001$ & $48[40,57]$ & 0.005 & 0.55 \\
\hline $\operatorname{LAEF}(\%)$ & $57[54,29]$ & $53[47,58]$ & 0.15 & $55[48,59]$ & 0.24 & 0.37 \\
\hline \multicolumn{7}{|l|}{ Pressure gradients } \\
\hline $\begin{array}{l}\text { Peak gradient Ao } \\
\text { (mmHg)* }\end{array}$ & NA & $85[72,107]$ & NA & $23[14,32]$ & 0.005 & NA \\
\hline $\begin{array}{l}\text { Mean gradient Ao } \\
(\mathrm{mmHg})^{*}\end{array}$ & NA & $49[42,62]$ & NA & $11[7,15]$ & 0.005 & NA \\
\hline \multicolumn{7}{|c|}{ Cardiopulmonary exercise test } \\
\hline $\begin{array}{l}\text { Peak VO2 } \\
\qquad\left(\mathrm{L} \mathrm{min}^{-1}\right)\end{array}$ & NA & $1.98[1.36,2.61]$ & NA & $2.21[1.52,2.74]$ & 0.06 & NA \\
\hline $\begin{array}{l}\text { Exercise capacity } \\
\text { (Watt) }\end{array}$ & NA & $155[89,216]$ & NA & $161[97,224]$ & 0.008 & NA \\
\hline
\end{tabular}

Data is presented as median (interquartile range)

$C O$ cardiac output, $E D V$ end-diastolic volume, $E S V$ end-systolic volume, $A V R$ aortic valve replacement, $A V S$ aortic valve stenosis, $L G E$ late gadolinium enhancement, $L V M$ LV mass, $L A E F$ left atrial emptying fraction, $L V E F$ LV ejection fraction, POST after surgical therapy, PRE before surgical therapy, $S V$ stroke volume, $V O 2$ oxygen consumption

* Measured by Doppler echocardiography

to $11[7,15] \mathrm{mmHg}, p=0.005$, respectively). Global LV dimensions and LA volume decreased after AVR, however there was no significant change in LVEF and LAEF (Table 2). Lateral wall thickening significantly improved after AVR and septal wall thickening showed a trend towards improvement albeit non-significant compared to before AVR (Table 3; Fig. 1 Supplemental Material). Both global $(-14[-16,13]$ to $-17[-18,-14] \%, p=0.4)$ and regional circumferential strain did not significantly improve after surgery (Table 3). Global longitudinal strain improved after AVR therapy $(-20[-23,-20]$ to -22 $[-23,-20] \%, p=0.013$, Fig. 2), with no differences between septal and lateral segments (Table 1 Supplementary Material). The amount of LGE after surgery did not change. Nonetheless, exercise capacity during cardiopulmonary exercise test showed a significant improvement from 155 [89, 216] to $161[97,224]$ Watt, $p=0.008$. Peak VO2 only showed a trend toward improvement (1.98 [1.36, 2.61] vs. $\left.2.21[1.52,2.74] \mathrm{L} \mathrm{min}^{-1}, p=0.06\right)$.

\section{Effect on myocardial function after septal myectomy}

From a total of ten HOCM patients, one patient declined follow up CMR, and another patient underwent pacemaker implantation due to an atrioventricular block. Measurements at baseline were included from ten patients, and at followup from the remaining eight patients. Both LVOT peak and mean gradients improved after septal myectomy (from $26[15,54]$ to $5[3,9] \mathrm{mmHg}$ and $14[8,23]$ to $8[5,19]$ $\mathrm{mmHg}, p=0.02$, respectively). As was to be expected, septal EDWT decreased following myectomy. Also, LV mass and LA dimension decreased after myectomy, but without a change in LV dimensions, LVEF or LAEF (Table 4). Although wall thickening showed a non-significant difference after myectomy (Fig. 1 Supplementary Material), global circumferential strain did not improve after myectomy $(-13[-16,-11]$ to $-14[-15,-11] \%, p=0.52)$. With respect to regional circumferential strain only septal circumferential strain further deteriorated with worsening of septal systolic strain rate (Table 5). Additionally global and 
Table 3 Aortic valve stenosis before (PRE) and after (POST) aortic valve replacement: regional characteristics

\begin{tabular}{|c|c|c|c|c|c|c|c|}
\hline & & Controls $(n=14)$ & $\begin{array}{l}\text { AVS pre-AVR } \\
(\mathrm{n}=10)\end{array}$ & $\begin{array}{l}p \text {-value controls } \\
\text { versus AVS pre- } \\
\text { AVR }\end{array}$ & $\begin{array}{l}\text { AVS post-AVR } \\
(\mathrm{n}=10)\end{array}$ & $\begin{array}{l}p \text {-value AVS } \\
\text { pre-AVR versus } \\
\text { AVS post-AVR }\end{array}$ & $\begin{array}{l}p \text {-value AVS } \\
\text { post-AVR versus } \\
\text { controls }\end{array}$ \\
\hline \multirow[t]{6}{*}{ Septal wall } & $\begin{array}{l}\text { ED wall thick- } \\
\text { ness (mm) }\end{array}$ & $6[6,7]$ & $11[11,13]$ & $<0.001$ & $9[8,10]$ & 0.005 & $<0.001$ \\
\hline & $\begin{array}{l}\text { Wall thickening } \\
(\%)\end{array}$ & $80[64,97]$ & $37[33,59]$ & 0.001 & $54[43,74]$ & 0.10 & 0.013 \\
\hline & \multicolumn{7}{|c|}{ Circumferential strain } \\
\hline & $\begin{array}{l}\text { Peak circum- } \\
\text { ferential } \\
\text { strain }(\%)\end{array}$ & $-17[-18,-14]$ & $-14[-16,-12]$ & 0.013 & $\begin{array}{l}-16[-18 \\
-13]\end{array}$ & 0.11 & 0.37 \\
\hline & $\begin{array}{l}\text { Peak systolic } \\
\text { circumferen- } \\
\text { tial strain rate } \\
\left(\% \mathrm{~s}^{-1}\right)\end{array}$ & $-47[-58,-43]$ & $-34[-44,-27]$ & 0.005 & $\begin{array}{l}-40[-53, \\
-32]\end{array}$ & 0.20 & 0.14 \\
\hline & $\begin{array}{l}\text { Peak diastolic } \\
\text { circumferen- } \\
\text { tial strain rate } \\
\left(\% \mathrm{~s}^{-1}\right)\end{array}$ & $36[26,39]$ & $27[12,43]$ & 0.29 & $26[22,42]$ & $0.24^{\mathrm{a}}$ & 0.57 \\
\hline \multirow[t]{6}{*}{ Lateral wall } & $\begin{array}{l}\text { ED wall thick- } \\
\text { ness }(\mathrm{mm})\end{array}$ & $6[5,6]$ & $10[9,11]$ & $<0.001$ & $9[8,9]$ & 0.01 & $<0.001$ \\
\hline & $\begin{array}{l}\text { Wall thickening } \\
(\%)\end{array}$ & $91[77,110]$ & $31[27,52]$ & $<0.001$ & $54[38,77]$ & 0.01 & 0.01 \\
\hline & \multicolumn{7}{|c|}{ Circumferential strain } \\
\hline & $\begin{array}{l}\text { Peak circum- } \\
\text { ferential } \\
\text { strain }(\%)\end{array}$ & $-17[-19,-13]$ & $-16[-21,-10]$ & 0.84 & $-16[-21,-12]$ & 0.88 & 0.98 \\
\hline & $\begin{array}{l}\text { Peak systolic } \\
\text { circumferen- } \\
\text { tial strain rate } \\
\left(\% \mathrm{~s}^{-1}\right)\end{array}$ & $-37[-50,-26]$ & $-50[-72,-30]$ & 0.24 & $-45[-51,-38]$ & 0.46 & 0.19 \\
\hline & $\begin{array}{l}\text { Peak diastolic } \\
\text { circumferen- } \\
\text { tial strain rate } \\
\left(\% \mathrm{~s}^{-1}\right)\end{array}$ & $13[10,18]$ & $17[13,26]$ & 0.13 & $18[12,25]$ & $0.35^{\mathrm{a}}$ & 0.19 \\
\hline
\end{tabular}

Data is presented as median (interquartile range)

$A V R$ aortic valve replacement, $A V S$ aortic valve stenosis, $E D$ end-diastolic, $P O S T$ after surgical therapy, PRE before surgical therapy

${ }^{a}$ Due to tagfading two patients were excluded from lateral diastolic strain rate analysis

regional longitudinal strain did not improve after myectomy (Fig. 3; Table 1 Supplementary Material). The amount of late gadolinium enhancement showed no significant increase after myectomy. Similar to AVS, septal myectomy showed a significant increase in exercise capacity during cardiopulmonary exercise test $(140$ [105, 170] vs. 152 [121, 221] Watt, $p=0.043)$, while peak VO2 remained similar (1.89 [1.26, $2.05]$ vs. $\left.1.92[1.50,2.79] \mathrm{L} \mathrm{min}^{-1}, p=0.89\right)$.

\section{Difference in myocardial adaptation after aortic valve replacement and septal myectomy}

In both patient groups, there was a significant reduction in intraventricular pressure gradients with a concomitant decrease in LA volume ( $p<0.01)$, suggesting lowering of diastolic filling pressures. Wall thickening improved after AVR, albeit without improvement in strain and strain rates. Similar to AVS patients, strain rates of the lateral wall were not affected after surgery for HOCM, while septal systolic circumferential strain rate further deteriorated, probably as a consequence of the myectomy. Both AVS and HOCM patients showed a reduction in LV wall thickness and mass after surgery, though this effect was more pronounced in AVS patients ( $p<0.05$, Fig. 3). Interestingly, both patient groups had an improvement in exercise capacity, most likely associated to the relieve in outflow obstruction. 


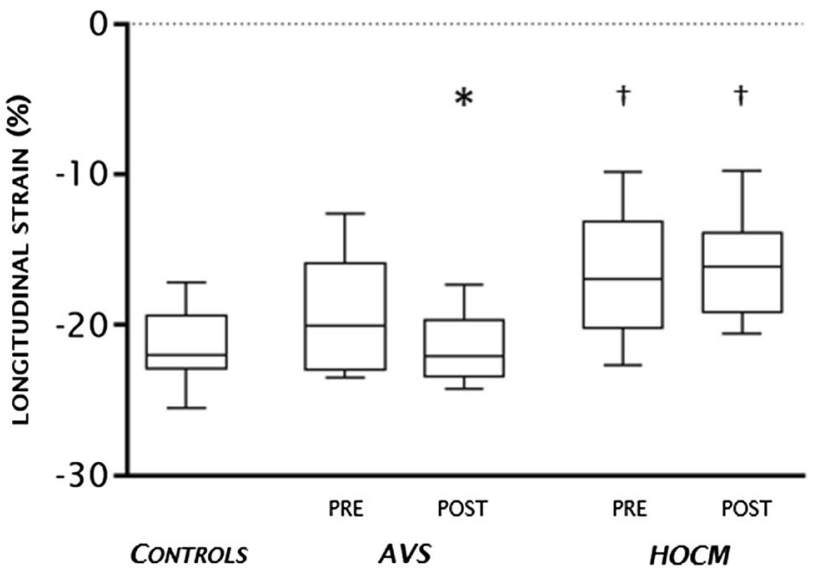

Fig. 2 Global longitudinal strain. Global longitudinal strain is depicted for AVS before (pre) and after (post) surgery, HOCM before (pre) and after (post) surgery and healthy controls. AVS patients showed similar longitudinal strain compared with healthy controls both before and after AVR therapy. HOCM patients revealed a significantly lower longitudinal strain compared with healthy controls even after myecomy. $\downarrow p<0.01$ versus controls. ${ }^{*} p<0.05$ follow up versus baseline. All data is presented by median with interquartile range. $A V S$ aortic valve stenosis; HOCM hypertrophic obstructive cardiomyopathy

\section{Discussion}

This study provided the unique opportunity to prospectively investigate myocardial function in patients with two different etiologies in LVH, and to what extent reverse remodeling occurs in AVS and HOCM patients after surgical treatment, AVR and septal myectomy respectively. The main findings of the present study are that (1) both AVS and HOCM patients have significantly higher LV mass and LA volumes than controls, and in HOCM smaller LV volumes compared with AVS patients and controls; (2) both patient groups had lower septal wall thickening and systolic strain rates than controls; (3) both patient groups demonstrated reversed structural remodeling after surgery, with improved intraventricular pressure gradients and a concomitant decrease in LA volume, decreased wall thickness and LV mass; (4) only AVS patients showed functional improvement after surgical treatment, evident from improved global wall thickening but unaffected strain rates, whereas HOCM patients deteriorated with respect to septal wall thickening and systolic strain rate.

In both patient groups, intraventricular pressure gradients improved after surgery with a concomitant reduced LA volume, probably reflecting improved diastolic pressures.

Table 4 Hypertrophic obstructive cardiomyopathy before (PRE) versus after (POST) septal myectomy: global characteristics

\begin{tabular}{|c|c|c|c|c|c|c|}
\hline & Controls $(n=14)$ & $\begin{array}{l}\text { HOCM pre- } \\
\text { myectomy } \\
(\mathrm{n}=10)\end{array}$ & $\begin{array}{l}p \text {-value controls vs } \\
\text { HOCM pre-myec- } \\
\text { tomy }\end{array}$ & $\begin{array}{l}\text { HOCM post- } \\
\text { myectomy } \\
(\mathrm{n}=8)\end{array}$ & $\begin{array}{l}p \text {-value HOCM } \\
\text { pre-myectomy } \\
\text { versus HOCM post- } \\
\text { myectomy }\end{array}$ & $\begin{array}{l}p \text {-value HOCM post- } \\
\text { myectomy versus } \\
\text { controls }\end{array}$ \\
\hline \multicolumn{7}{|c|}{ Global LV characteristics } \\
\hline $\operatorname{LV} \operatorname{EDV}\left(\mathrm{ml} \mathrm{m}^{-2}\right)$ & $90[81,104]$ & $93[78,104]$ & 0.93 & $76[67,96]$ & 0.16 & 0.21 \\
\hline $\operatorname{LV} \operatorname{ESV}\left(\mathrm{ml} \mathrm{m}^{-2}\right)$ & $36[26,40]$ & $24[21,31]$ & 0.02 & $25[20,29]$ & 0.67 & 0.24 \\
\hline LVEF (\%) & $61[57,66]$ & $73[65,74]$ & 0.009 & $66[61,73]$ & 0.21 & 0.13 \\
\hline $\mathrm{SV}(\mathrm{ml})$ & $114[101,124]$ & $133[92,166]$ & 0.34 & $112[91,146]$ & 0.21 & 0.92 \\
\hline $\mathrm{CO}\left(\mathrm{L} \mathrm{min}^{-1}\right)$ & $7.9[6.5,8.8]$ & $7.6[2.8,10]$ & 0.55 & $8.4[7.3,9.5]$ & 0.26 & 0.53 \\
\hline $\operatorname{LVM}\left(\mathrm{g} \mathrm{m}^{-2}\right)$ & $50[44,54]$ & $92[90,114]$ & $<0.001$ & $81[67,91]$ & 0.03 & $<0.001$ \\
\hline LGE mass (\%) LV & 0 & $4.0[1.7,11.3]$ & NA & $4.9[1.1,36.4]$ & 0.88 & NA \\
\hline \multicolumn{7}{|l|}{ LA characteristics } \\
\hline $\begin{array}{l}\text { LA volume (ml } \\
\left.\mathrm{m}^{-2}\right)\end{array}$ & $45[42,50]$ & $84[69,114]$ & $<0.001$ & $62[51,80]$ & 0.012 & $<0.001$ \\
\hline LAEF (\%) & $57[54,29]$ & $41[27,46]$ & $<0.001$ & $46[42,54]$ & 0.16 & 0.002 \\
\hline \multicolumn{7}{|l|}{ Pressure gradients } \\
\hline $\begin{array}{l}\text { Peak gradient } \\
\text { LVOT }(\mathrm{mmHg}) *\end{array}$ & NA & $14[8,23]$ & NA & $8[5,19]$ & 0.02 & NA \\
\hline $\begin{array}{l}\text { Mean gradient } \\
\text { LVOT }(\mathrm{mmHg}) *\end{array}$ & NA & $26[15,54]$ & NA & $5[3,9]$ & 0.02 & NA \\
\hline \multicolumn{7}{|c|}{ Cardiopulmonary exercise test } \\
\hline $\begin{array}{l}\text { Peak VO2 (L } \\
\left.\min ^{-1}\right)\end{array}$ & NA & $1.89[1.26,2.05]$ & NA & $1.92[1.50,2.79]$ & 0.89 & NA \\
\hline $\begin{array}{l}\text { Exercise capacity } \\
\text { (Watt) }\end{array}$ & NA & $140[105,170]$ & NA & $152[121,221]$ & 0.043 & NA \\
\hline
\end{tabular}

Data is presented as median (interquartile range)

HOCM hypertrophic obstructive cardiomyopathy; other abbreviations as in Table 2 
Table 5 Hypertrophic obstructive cardiomyopathy before (PRE) versus after (POST) septal myectomy: regional characteristics

\begin{tabular}{|c|c|c|c|c|c|c|c|}
\hline & & Controls $(n=14)$ & $\begin{array}{l}\text { HOCM pre- } \\
\text { myectomy } \\
(n=10)\end{array}$ & $\begin{array}{l}p \text {-value controls } \\
\text { versus HOCM } \\
\text { pre-myectomy }\end{array}$ & $\begin{array}{l}\text { HOCM post- } \\
\text { myectomy } \\
(\mathrm{n}=8)\end{array}$ & $\begin{array}{l}p \text {-value HOCM } \\
\text { pre-myectomy } \\
\text { versus post- } \\
\text { myectomy }\end{array}$ & $\begin{array}{l}p \text {-value HOCM } \\
\text { post-myectomy } \\
\text { versus controls }\end{array}$ \\
\hline \multirow[t]{6}{*}{ Septal wall } & $\begin{array}{l}\text { ED wall thick- } \\
\text { ness }(\mathrm{mm})\end{array}$ & $6[6,7]$ & $15[13,17]$ & $<0.001$ & $13[10,16]$ & 0.04 & $<0.001$ \\
\hline & $\begin{array}{l}\text { Wall thickening } \\
(\%)\end{array}$ & $80[64,97]$ & $50[33,66]$ & 0.009 & $61[36,63]$ & 0.33 & 0.02 \\
\hline & \multicolumn{7}{|c|}{ Circumferential strain } \\
\hline & $\begin{array}{l}\text { Peak circumfer- } \\
\text { ential strain } \\
(\%)\end{array}$ & $-17[-18,-14]$ & $-10[-15,-9]$ & 0.003 & $-8[-10,-7]$ & 0.02 & $<0.001$ \\
\hline & $\begin{array}{l}\text { Peak systolic } \\
\text { circumferen- } \\
\text { tial strain rate } \\
\left(\% \mathrm{~s}^{-1}\right)\end{array}$ & $-47[-58,-43]$ & $-28[-44,-17]$ & 0.005 & $-13[-17,-6]$ & 0.03 & $<0.001$ \\
\hline & $\begin{array}{l}\text { Peak diastolic } \\
\text { circumferen- } \\
\text { tial strain rate } \\
\left(\% \mathrm{~s}^{-1}\right)\end{array}$ & $36[26,39]$ & $13[9,33]$ & 0.02 & $15[10,23]$ & $0.46 \mathrm{a}$ & 0.002 \\
\hline \multirow[t]{6}{*}{ Lateral wall } & $\begin{array}{l}\text { ED wall thick- } \\
\text { ness }(\mathrm{mm})\end{array}$ & $6[5,6]$ & $10[9,12]$ & $<0.001$ & $10[8,11]$ & 0.78 & $<0.001$ \\
\hline & $\begin{array}{l}\text { Wall thickening } \\
(\%)\end{array}$ & $91[77,110]$ & $83[49,128]$ & 0.71 & $98[60,123]$ & 0.26 & 0.97 \\
\hline & \multicolumn{7}{|c|}{ Circumferential strain } \\
\hline & $\begin{array}{l}\text { Peak circumfer- } \\
\text { ential strain } \\
(\%)\end{array}$ & $-17[-19,-13]$ & $-16[-19,-13]$ & 0.78 & $-19[-21,-16]$ & 0.17 & 0.22 \\
\hline & $\begin{array}{l}\text { Peak systolic } \\
\text { circumferen- } \\
\text { tial strain rate } \\
\left(\% \mathrm{~s}^{-1}\right)\end{array}$ & $-37[-50,-26]$ & $-46[-56,-32]$ & 0.48 & $-53[-64,-42]$ & 0.23 & 0.046 \\
\hline & $\begin{array}{l}\text { Peak diastolic } \\
\text { circumferen- } \\
\text { tial strain rate } \\
\left(\% \mathrm{~s}^{-1}\right)\end{array}$ & $13[10,18]$ & $15[11,22]$ & 0.44 & $17[11,22]$ & $0.92^{\mathrm{a}}$ & 0.40 \\
\hline
\end{tabular}

Data is presented as median (interquartile range)

HOCM hypertrophic cardiomyopathy; other abbreviations as in Table 4

${ }^{a}$ Due to tagfading two patients were excluded from diastolic strain rate analysis

AVR is known to improve symptoms, reduce LV mass and wall thickness [24, 25], which is in line with the results in our cohort. Whereas Staron and colleagues demonstrated improvement in echocardiographic circumferential strain after AVR [26], in our cohort we found an even greater improvement comparable to normal values, suggesting that AVR reversibly affects the LV. A previous study using myocardial tagging showed global diastolic dysfunction before AVR and improvement after surgery [27]. This may be explained by the delayed and prolonged diastolic untwisting in AVS before AVR [28]. In this study AVS patients had normal diastolic function before AVR similar to controls, probably due to an earlier timing of the surgical intervention. While there is still debate to advise betablockers in order to reduce afterload in AVS patients [3], in our cohort none of the patients used betablockers. Taken together these findings confirm the benefit of decreasing the pressure gradient by valve replacement, thereby restoring structure and function and ultimately improving exercise capacity.

Whereas the increased pressure gradient in AVS is a static phenomenon, the pressure gradient in HOCM is of a dynamic nature, mainly depending on loading conditions [16]. Septal myectomy and alcohol ablation are both equally effective at reducing LVOT obstruction, however, septal myectomy is shown to be more effective in improving exercise parameters and results in a consistent septum reduction [29]. This study demonstrated a reduction in LV mass and septal wall thickness which is in line with previous results [30]. Although myectomy demonstrated reversed structural remodeling at the septum, surgery may have induced 


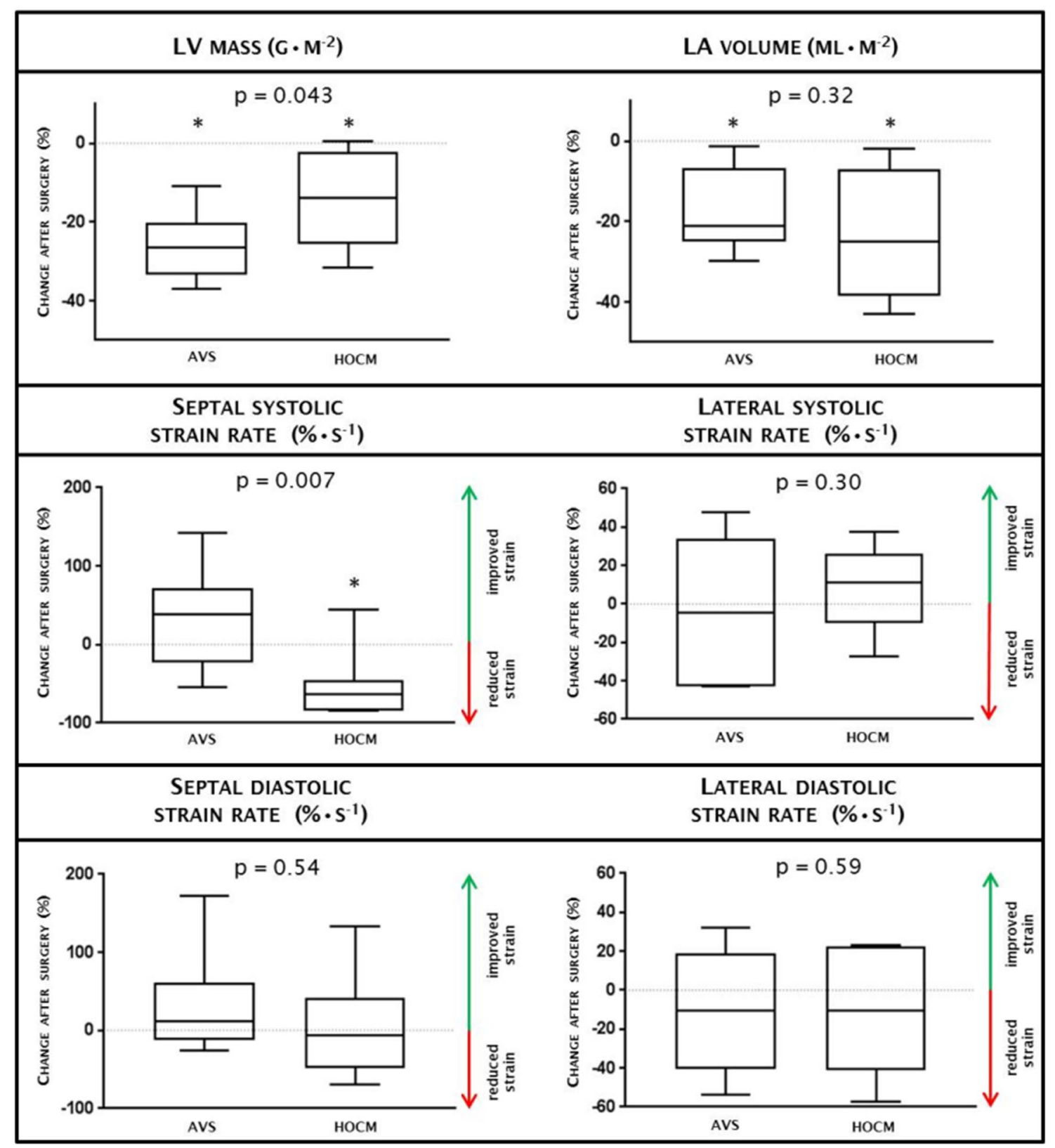

Fig. 3 Change after surgery between AVS and HOCM. Change after surgery between AVS $(n=10)$ and HOCM $(n=8)$ are depicted for LV myocardial mass, left atrial volume, circumferential strain and regional systolic and diastolic stain rates. Values above zero in strain rates indicate improved strain, values below zero indicate reduced strain. An asterisk $\left(^{*}\right)$ indicates significant change within AVS or HOCM before vs after surgery ( $p$-values are mentioned in Tables 2,

myocardial dysfunction. Global dysfunction of circumferential strain was also seen by Moravsky et al. by echocardiography [10], which is in agreement with our measurements by CMR. Regional analysis demonstrated impaired septal systolic and diastolic function before surgery, whereas the lateral wall showed similar values compared to healthy controls. The differences between septum and lateral wall may
4). All data is presented by median with interquartile range. After surgery, HOCM demonstrates deterioration of septal systolic strain rate compared with AVS. Changes after surgery in regional diastolic strain rates were similar in HOCM compared to AVS. AVS aortic valve stenosis, HOCM hypertrophic obstructive cardiomyopathy, $L A$ left atrial, $L V$ left ventricular

be explained by the difference of tissue characteristics. At the hypertrophied septum tissue characteristics demonstrate increased amount of extracellular volume and myocardial disarray compared with the lateral wall. These myocardial differences are in accordance with a longitudinal study in HOCM patients after alcohol septum ablation, in which septal systolic function further deteriorated after intervention 
[11]. Sommer et al. demonstrated reduced longitudinal strain up to 3 years after alcohol septal ablation in HOCM patients [31]. The current study also demonstrated reduced longitudinal strain even after myectomy, while contrast enhancement was similar compared to before myectomy [32]. In contrast to septal alcohol ablation therapy in HOCM patients, where a myocardial infarction is induced to achieve septal reduction which leads to increased scarring [11]. In our HOCM population scar is not responsible for the functional deterioration after myectomy. This implies that other mechanisms may be responsible for this loss in function, such as loss of myocyte integrity or progression of myocyte disarray [33]. In addition, the presence of sarcomere mutations continue to cause inhomogeneous contraction of the sarcomeres in the remaining cardiomyocytes after septal myectomy and consequently further reduce myocardial function. Although myectomy did not improve regional function in our population, a recent study demonstrated that myectomy in HOCM patients had a positive effect on the incidence of sudden cardiac death and implantable cardioverter-defibrillator discharge [34]. However, to be able to better understand and define the pathological process of functional loss of the myocardium, future studies using new imaging techniques, such as extracellular volume fraction assessment using T1 mapping, may be useful [35].

Diastolic dysfunction is a hallmark of HOCM, and seems to be largely caused by increased interstitial fibrosis leading to reduced LV compliance [36]. The reduction in LA volume after myectomy in our population suggests an improvement in diastolic intraventricular pressures, this study however did not demonstrate improvement in global or regional diastolic function, which might be explained by the reduced LV compliance [37]. Accordingly, an echocardiographic study also revealed reduction in LA volume without improvement in diastolic function and might be related to the disease history and increased development of interstitial fibrosis [12]. Summarizing, our findings we demonstrate reversed structural remodeling after myectomy, and worsening of functional remodeling at the septum. Even though functional remodeling deteriorated after surgery, HOCM patients managed to improve exercise capacity, which seems to be a direct result of relieving the LVOT obstruction by myectomy [29].

The present study demonstrates reversed structural remodeling in both AVS and HOCM patients after surgery, however, recovery in systolic function was only seen in AVS. Furthermore, HOCM patients demonstrated systolic functional deterioration which seems to be inherent to septal myectomy and the ongoing and irreversible cardiac pathophysiology. Although septal myectomy reduces the risk of sudden cardiac death, this study emphasizes the need for future research in therapies to enhance myocardial recovery.

However, there are several limitation in this study. Several medical centers were involved in the inclusion of patients for advanced imaging before and after cardiac surgery, and therefore, the included patients do not reflect the actual number of patients who yearly undergo cardiac surgery. As the number of participants included were limited, the conclusions should be interpreted carefully. Yet, even with this relative small study population we were able to demonstrate differences in myocardial adaptation after surgery for AVS and HOCM. Although tissue tagging is a novel and accurate method to assess myocardial function, tagline fading in end-diastole occurred in two patients who were therefore excluded from the diastolic strain rate analysis. Accurate detection of diffuse fibrosis using T1 mapping might have increased our understanding of the absence of functional recovery in HOCM. Furthermore, evaluation by CMR was performed at 4 months after surgery which seems reasonable but not necessarily the optimal timing to capture full remodeling and functional recovery.

Funding This study was funded by the 7th Framework Program of the European Union ('BIGHEART', Grant agreement 241577), from the Netherlands organization for scientific research (NWO; VIDI Grant 917.11.344), the Netherlands Heart Foundation ('Dekker Grant' Grant agreement 2011T33) and Netherlands Cardiovascular Research Initiative: An initiative supported by the Netherlands Heart Foundation, CVON2014-40 DOSIS.

\section{Compliance with ethical standards}

Conflict of interest The authors declare that they have no conflict of interest.

Ethical approval All procedures performed in studies involving human participants were in accordance with the ethical standards of the institutional and/or national research committee and with the 1964 Helsinki declaration and its later amendments or comparable ethical standards.

Informed consent Informed consent was obtained from all individual participants included in the study.

Open Access This article is distributed under the terms of the Creative Commons Attribution 4.0 International License (http://creativeco mmons.org/licenses/by/4.0/), which permits unrestricted use, distribution, and reproduction in any medium, provided you give appropriate credit to the original author(s) and the source, provide a link to the Creative Commons license, and indicate if changes were made.

\section{References}

1. Gavina C, Falcao-Pires I, Rocha-Goncalves F, Leite-Moreira A (2012) Left ventricular hypertrophy in isolated aortic stenosis: primetime for the ventricle. Curr Pharm Biotechnol 13(13):2503-2514

2. Rosenhek R, Zilberszac R, Schemper M, Czerny M, Mundigler G, Graf S, Bergler-Klein J, Grimm M, Gabriel H, Maurer G (2010) Natural history of very severe aortic stenosis. 
Circulation 121(1):151-156. https://doi.org/10.1161/CIRCU LATIONAHA.109.894170

3. Vahanian A, Baumgartner H, Bax J, Butchart E, Dion R, Filippatos G, Flachskampf F, Hall R, Iung B, Kasprzak J, Nataf P, Tornos P, Torracca L, Wenink A (2007) Guidelines on the management of valvular heart disease: the task force on the management of valvular heart disease of the european society of cardiology. Eur Heart J 28(2):230-268. https://doi.org/10.1093/eurheartj/eh1428

4. Semsarian C, Ingles J, Maron MS, Maron BJ (2015) New perspectives on the prevalence of hypertrophic cardiomyopathy. J Am Coll Cardiol 65(12):1249-1254. https://doi.org/10.1016/j. jacc.2015.01.019

5. Kirschner SE, Becker E, Antognozzi M, Kubis HP, Francino A, Navarro-Lopez F, Bit-Avragim N, Perrot A, Mirrakhimov MM, Osterziel KJ, McKenna WJ, Brenner B, Kraft T (2005) Hypertrophic cardiomyopathy-related beta-myosin mutations cause highly variable calcium sensitivity with functional imbalances among individual muscle cells. Am J Physiol Heart Circ Physiol 288(3):H1242-H1251. https://doi.org/10.1152/ajpheart.00686 .2004

6. Ashrafian H, Redwood C, Blair E, Watkins H (2003) Hypertrophic cardiomyopathy:a paradigm for myocardial energy depletion. Trends Genet 19(5):263-268. https://doi.org/10.1016/S0168 -9525(03)00081-7

7. Abraham MR, Bottomley PA, Dimaano VL, Pinheiro A, Steinberg A, Traill TA, Abraham TP, Weiss RG (2013) Creatine kinase adenosine triphosphate and phosphocreatine energy supply in a single kindred of patients with hypertrophic cardiomyopathy. Am J Cardiol 112(6):861-866. https://doi.org/10.1016/j.amjca rd.2013.05.017

8. Maron MS, Olivotto I, Betocchi S, Casey SA, Lesser JR, Losi MA, Cecchi F, Maron BJ (2003) Effect of left ventricular outflow tract obstruction on clinical outcome in hypertrophic cardiomyopathy. N Engl J Med 348(4):295-303. https://doi.org/10.1056/NEJMo a021332

9. McLeod CJ, Ommen SR, Ackerman MJ, Weivoda PL, Shen WK, Dearani JA, Schaff HV, Tajik AJ, Gersh BJ (2007) Surgical septal myectomy decreases the risk for appropriate implantable cardioverter defibrillator discharge in obstructive hypertrophic cardiomyopathy. Eur Heart J 28(21):2583-2588. https://doi.org/10.1093/ eurheartj/ehm117

10. Moravsky G, Bruchal-Garbicz B, Jamorski M, Ralph-Edwards A, Gruner C, Williams L, Woo A, Yang H, Laczay B, Rakowski H, Carasso S (2013) Myocardial mechanical remodeling after septal myectomy for severe obstructive hypertrophic cardiomyopathy. J Am Soc Echocardiogr 26(8):893-900. https://doi.org/10.1016/j. echo.2013.05.012

11. van Dockum WG, Kuijer JP, Gotte MJ, Ten Cate FJ, Ten Berg JM, Beek AM, Twisk JW, Marcus JT, Visser CA, van Rossum AC (2006) Septal ablation in hypertrophic obstructive cardiomyopathy improves systolic myocardial function in the lateral (free) wall: a follow-up study using CMR tissue tagging and 3D strain analysis. Eur Heart J 27(23):2833-2839. https://doi.org/10.1093/ eurheartj/ehl358

12. Finocchiaro G, Haddad F, Kobayashi Y, Lee D, Pavlovic A, Schnittger I, Sinagra G, Magavern E, Myers J, Froelicher V, Knowles JW, Ashley E (2016) Impact of septal reduction on left atrial size and diastole in hypertrophic cardiomyopathy. Echocardiography 33(5):686-694. https://doi.org/10.1111/echo.13158

13. Fairbairn TA, Steadman CD, Mather AN, Motwani M, Blackman DJ, Plein S, McCann GP, Greenwood JP (2013) Assessment of valve haemodynamics, reverse ventricular remodelling and myocardial fibrosis following transcatheter aortic valve implantation compared to surgical aortic valve replacement: a cardiovascular magnetic resonance study. Heart 99(16):1185-1191. https://doi. org/10.1136/heartjnl-2013-303927

14. Guclu A, Germans T, Witjas-Paalberends ER, Stienen GJ, Brouwer WP, Harms HJ, Marcus JT, Vonk AB, Stooker W, Yilmaz A, Klein P, Ten Berg JM, Kluin J, Asselbergs FW, Lammertsma AA, Knaapen P, van Rossum AC, van der Velden J (2013) ENerGetIcs in hypertrophic cardiomyopathy: translation between MRI, PET and cardiac myofilament function (ENGINE study). Neth Heart J 21(12):567-571. https://doi.org/10.1007/s12471-013-0478-8

15. Cheitlin MD, Armstrong WF, Aurigemma GP, Beller GA, Bierman FZ, Davis JL, Douglas PS, Faxon DP, Gillam LD, Kimball TR, Kussmaul WG, Pearlman AS, Philbrick JT, Rakowski H, Thys DM, Antman EM, Smith SC Jr, Alpert JS, Gregoratos G, Anderson JL, Hiratzka LF, Hunt SA, Fuster V, Jacobs AK, Gibbons RJ, Russell RO (2003) ACC/AHA/ASE 2003 guideline update for the clinical application of echocardiography: summary article: a report of the American College of Cardiology/American Heart Association task force on practice guidelines (ACC/AHA/ASE Committee to Update the 1997 guidelines for the clinical application of echocardiography). Circulation 108(9):1146-1162. https ://doi.org/10.1161/01.cir.0000073597.57414.a9

16. Elliott PM, Anastasakis A, Borger MA, Borggrefe M, Cecchi F, Charron P, Hagege AA, Lafont A, Limongelli G, Mahrholdt H, McKenna WJ, Mogensen J, Nihoyannopoulos P, Nistri S, Pieper PG, Pieske B, Rapezzi C, Rutten FH, Tillmanns C, Watkins H (2014) 2014 ESC Guidelines on diagnosis and management of hypertrophic cardiomyopathy: the task force for the diagnosis and management of hypertrophic cardiomyopathy of the European Society of Cardiology (ESC). Eur Heart J 35(39):2733-2779. https://doi.org/10.1093/eurheartj/ehu284

17. Baumgartner H, Falk V, Bax JJ, De Bonis M, Hamm C, Holm PJ, Iung B, Lancellotti P, Lansac E, Rodriguez Munoz D, Rosenhek R, Sjogren J, Tornos Mas P, Vahanian A, Walther T, Wendler O, Windecker S, Zamorano JL, Group ESCSD (2017) 2017 ESC/ EACTS guidelines for the management of valvular heart disease. Eur Heart J 38(36):2739-2791. https://doi.org/10.1093/eurheartj/ ehx391

18. Germans T, Gotte MJ, Nijveldt R, Spreeuwenberg MD, Beek AM, Bronzwaer JG, Visser CA, Paulus WJ, van Rossum AC (2007) Effects of aging on left atrioventricular coupling and left ventricular filling assessed using cardiac magnetic resonance imaging in healthy subjects. Am J Cardiol 100(1):122-127. https://doi. org/10.1016/j.amjcard.2007.02.060

19. Zwanenburg JJ, Kuijer JP, Marcus JT, Heethaar RM (2003) Steady-state free precession with myocardial tagging: CSPAMM in a single breathhold. Magn Reson Med 49(4):722-730. https:// doi.org/10.1002/mrm. 10422

20. Creatis inTag, CMRSegTools and CMRDiffTools. A suite of tools for analysing cardiac MR images

21. Swoboda PP, Larghat A, Zaman A, Fairbairn TA, Motwani M, Greenwood JP, Plein S (2014) Reproducibility of myocardial strain and left ventricular twist measured using complementary spatial modulation of magnetization. J Magn Reson Imaging 39(4):887-894. https://doi.org/10.1002/jmri.24223

22. Arts T, Prinzen FW, Delhaas T, Milles JR, Rossi AC, Clarysse P (2010) Mapping displacement and deformation of the heart with local sine-wave modeling. IEEE Trans Med Imaging 29(5):11141123. https://doi.org/10.1109/tmi.2009.2037955

23. Cerqueira MD, Weissman NJ, Dilsizian V, Jacobs AK, Kaul S, Laskey WK, Pennell DJ, Rumberger JA, Ryan T, Verani MS (2002) Standardized myocardial segmentation and nomenclature for tomographic imaging of the heart. A statement for healthcare professionals from the Cardiac Imaging Committee of the Council on clinical cardiology of the American Heart Association. Circulation 105(4):539-542 
24. Garcia-Villarreal O, Heredia-Delgado JA, Ramirez-Gonzalez B, Saldana-Becerra MA, Gonzalez-Alanis MA, Garcia-Guevara MI, Sanchez-Sanchez LM (2016) Left ventricular adaptive response after surgery of aortic valve replacement for severe valvular stenosis. Gac Med Mex 152(2):191-195

25. Treibel TA, Kozor R, Schofield R, Benedetti G, Fontana M, Bhuva AN, Sheikh A, Lopez B, Gonzalez A, Manisty C, Lloyd G, Kellman P, Diez J, Moon JC (2018) Reverse myocardial remodeling following valve replacement in patients with aortic stenosis. J Am Coll Cardiol 71(8):860-871. https://doi.org/10.1016/j. jacc.2017.12.035

26. Staron A, Bansal M, Kalakoti P, Nakabo A, Gasior Z, Pysz P, Wita K, Jasinski M, Sengupta PP (2013) Speckle tracking echocardiography derived 2-dimensional myocardial strain predicts left ventricular function and mass regression in aortic stenosis patients undergoing aortic valve replacement. Int J Cardiovasc Imaging 29(4):797-808. https://doi.org/10.1007/s10554-012-0160-z

27. Mahmod M, Bull S, Suttie JJ, Pal N, Holloway C, Dass S, Myerson SG, Schneider JE, De Silva R, Petrou M, Sayeed R, Westaby S, Clelland C, Francis JM, Ashrafian H, Karamitsos TD, Neubauer S (2013) Myocardial steatosis and left ventricular contractile dysfunction in patients with severe aortic stenosis. Circ Cardiovasc Imaging 6(5):808-816. https://doi.org/10.1161/circimagin g.113.000559

28. van Dalen BM, Tzikas A, Soliman OI, Kauer F, Heuvelman HJ, Vletter WB, ten Cate FJ, Geleijnse ML (2011) Left ventricular twist and untwist in aortic stenosis. Int J Cardiol 148(3):319-324. https://doi.org/10.1016/j.ijcard.2009.11.022

29. Firoozi S, Elliott PM, Sharma S, Murday A, Brecker SJ, Hamid MS, Sachdev B, Thaman R, McKenna WJ (2002) Septal myotomy-myectomy and transcoronary septal alcohol ablation in hypertrophic obstructive cardiomyopathy. A comparison of clinical, haemodynamic and exercise outcomes. Eur Heart $\mathbf{J}$ 23(20):1617-1624

30. Maron BJ, Maron MS (2013) Hypertrophic cardiomyopathy. Lancet 381(9862):242-255. https://doi.org/10.1016/S0140 $-6736(12) 60397-3$

31. Sommer A, Poulsen SH, Mogensen J, Thuesen L, Egeblad H (2010) Left ventricular longitudinal systolic function after alcohol septal ablation for hypertrophic obstructive cardiomyopathy: a long-term follow-up study focused on speckle tracking echocardiography. Eur J Echocardiogr 11(10):883-888. https://doi. org/10.1093/ejechocard/jeq087

32. Guclu A, Knaapen P, Harms HJ, Parbhudayal RY, Michels M, Lammertsma AA, van Rossum AC, Germans T, van der Velden
J (2017) Disease stage-dependent changes in cardiac contractile performance and oxygen utilization underlie reduced myocardial efficiency in human inherited hypertrophic cardiomyopathy. Circ Cardiovasc Imaging. https://doi.org/10.1161/CIRCIMAGIN G.116.005604

33. Dhillon A, Sweet W, Popovic ZB, Smedira NG, Thamilarasan M, Lytle BW, Tan C, Starling RC, Lever HM, Moravec CS, Desai MY (2014) Association of noninvasively measured left ventricular mechanics with in vitro muscle contractile performance: a prospective study in hypertrophic cardiomyopathy patients. J Am Heart Assoc 3(6):e001269. https://doi.org/10.1161/ JAHA.114.001269

34. Mentias A, Raeisi-Giglou P, Smedira NG, Feng K, Sato K, Wazni O, Kanj M, Flamm SD, Thamilarasan M, Popovic ZB, Lever HM, Desai MY (2018) Late gadolinium enhancement in patients with hypertrophic cardiomyopathy and preserved systolic function. J Am Coll Cardiol 72(8):857-870. https://doi.org/10.1016/j. jacc.2018.05.060

35. Kramer CM, Appelbaum E, Desai MY, Desvigne-Nickens P, DiMarco JP, Friedrich MG, Geller N, Heckler S, Ho CY, JeroschHerold M, Ivey EA, Keleti J, Kim DY, Kolm P, Kwong RY, Maron MS, Schulz-Menger J, Piechnik S, Watkins H, Weintraub WS, Wu $P$, Neubauer S (2015) Hypertrophic cardiomyopathy registry: the rationale and design of an international, observational study of hypertrophic cardiomyopathy. Am Heart J 170(2):223-230. https ://doi.org/10.1016/j.ahj.2015.05.013

36. Iles LM, Ellims AH, Llewellyn H, Hare JL, Kaye DM, McLean CA, Taylor AJ (2015) Histological validation of cardiac magnetic resonance analysis of regional and diffuse interstitial myocardial fibrosis. Eur Heart J Cardiovasc Imaging 16(1):14-22. https://doi. org/10.1093/ehjci/jeu182

37. Chen YZ, Qiao SB, Hu FH, Yuan JS, Yang WX, Cui JG, Zhang Y, Zhang CL (2015) Left ventricular remodeling and fibrosis: sex differences and relationship with diastolic function in hypertrophic cardiomyopathy. Eur J Radiol 84(8):1487-1492. https:// doi.org/10.1016/j.ejrad.2015.04.026

Publisher's Note Springer Nature remains neutral with regard to jurisdictional claims in published maps and institutional affiliations. 